\title{
Can a single model account for both risky choices and inter-temporal choices? Testing the assumptions underlying models of risky inter-temporal choice
}

\author{
Ashley Luckman ${ }^{1,2}$ - Chris Donkin ${ }^{1} \cdot$ Ben R. Newell ${ }^{1}$
}

Published online: 9 June 2017

(C) Psychonomic Society, Inc. 2017

\begin{abstract}
There is growing interest in modelling how people make choices that involve both risks and delays, i.e., risky inter-temporal choices. We investigated an untested assumption underlying several proposed risky inter-temporal choice models: that pure risky choices and pure inter-temporal choices are special cases of risky inter-temporal choice. We tested this assumption by presenting a single group of participants with risky choices and inter-temporal choices. We then compared the performance of a model that is fit to both choice types simultaneously, with the performance of separate models fit to the risky choice and inter-temporal choice data. We find, using Bayesian model comparison, that the majority of participants are best fit by a single model that incorporates both risky and inter-temporal choices. This result supports the assumption that risky choices and inter-temporal choices may be special cases of risky inter-temporal choice. Our results also suggest that, under the conditions of our experiment, interpretation of monetary value is very similar in risky choices and inter-temporal choices.
\end{abstract}

Keywords Risky choice $\cdot$ Inter-temporal choice $\cdot$ Utility

Electronic supplementary material The online version of this article (doi:10.3758/s13423-017-1330-8) contains supplementary material, which is available to authorized users.

Ashley Luckman

ashleyjames.luckman@unibas.ch

1 School of Psychology, University of New South Wales, Sydney, Australia

2 Center for Economic Psychology, Department of Psychology, University of Basel, Missionsstrasse 62a, 4055 Basel, Switzerland
Several recent papers have proposed models of decisions involving risky inter-temporal choices (Baucells \& Heukamp, 2010, 2012; Vanderveldt, Green \& Myerson, 2015; Yi, de la Piedad \& Bickel, 2006). These are choices that involve mixtures of probabilistic outcomes, such as a gamble with a $50 \%$ chance of receiving $\$ 100$, and delayed outcomes, such as having to wait several weeks or months to receive $\$ 100$. To date, three specific models have been proposed to deal with these choice types: the Probability and Time Trade-off model (Baucells \& Heukamp, 2010), the Multiplicative Hyperboloid Discounting model (Vanderveldt et al., 2015), and the Hyperbolic Discounting model of Yi and colleagues (2006). A common feature of these three models is that they treat pure risky choices and pure inter-temporal choices as special cases of risky intertemporal choices. In doing so, the models reduce to common variants of risky choice and inter-temporal choice models under particular conditions.

For instance, if all outcomes in a risky inter-temporal choice occur immediately - thereby making it a pure risky choice - all three models reduce to some form of Prospect Theory; one of the most popular models in the risky choice literature (Kahneman \& Tversky, 1979). Similarly, for any risky inter-temporal choice where all outcomes occur with certainty-a pure inter-temporal choice-both the Multiplicative Hyperboloid Discounting and Hyberbolic Discounting models reduce to their respective namesake inter-temporal choice models. The Probability and Time Trade-off Model reduces to what could be considered a modification of Ebert and Prelec's constant sensitivity function, another proposed inter-temporal choice model (see Doyle, 2013 for a review of inter-temporal choice models). Table 1 provides an example of a pure risky choice, a pure inter-temporal choice, and a typical risky inter-temporal choice. 
Table 1 Examples of four binary choices that involve risks, delays, or both

\begin{tabular}{lll}
\hline Choice type & Option 1 & Option 2 \\
\hline 1. Pure risky & $\$ 150$ with probability 0.9, else nothing & $\$ 225$ with probability 0.6, else nothing \\
2. Pure inter-temporal & $\$ 150$ in 2 months & $\$ 225$ in 9 months \\
3. Risky inter-temporal & $\$ 150$ in 2 months with probability 0.9, else nothing & $\$ 225$ in 9 months with probability 0.6, else nothing \\
4. Risk vs. delay & $\$ 150$ now with probability 0.9, else nothing & $\$ 225$ in 9 months for certain \\
\hline
\end{tabular}

Examples 1 and 2 involve only risk or delay. Example 3 represents a typical risky inter-temporal choice, whereas example 4 is a special subtype of risky inter-temporal choice

The purpose of this paper is to test the assumption that risky choices and inter-temporal choices are simply special cases of risky inter-temporal choices. The three existing models of risky inter-temporal choices are yet to be tested using pure risky or pure inter-temporal choices. For example, Baucells and Heukamp (2010) and Vanderveldt and colleagues (2015) tested their models with only risky inter-temporal choices, although Baucells and Heukamp included a small number of pure risky choices. Simplified versions of the Multiplicative Hyperboloid Discounting model have been fit to risk-only and delay-only choices, but never have both types of choices been fit by the same model or used explicit model selection techniques (Myerson, Green, Hanson, Holt \& Estle, 2003; Myerson, Green, Morris, 2011).

To test this crucial "reductionist" assumption, we used a dataset where the same participants made both pure risky and pure inter-temporal choices. We then compared the performance of a model fit to both choice types simultaneously, to the performance of separate models fit to the inter-temporal and risky choices. If we find that a single model fit to both choice types explains participants' data better-taking into account both the models fit to the data and its complexity - than using separate models to explain each choice type, then we have evidence that the reductionist assumption common to the three proposed risky inter-temporal choice models is justified. Such a finding would suggest that risky choices, inter-temporal choices, and risky inter-temporal choices are all made in a similar fashion. Furthermore, evidence for a single model would contribute to the ongoing debate regarding whether peoples' interpretation of monetary value is identical in both risky choices and inter-temporal choices (Abdellaoui, Bleichrodt, l'Haridon \& Paraschiv, 2013).

\section{Utility-based models}

The three aforementioned models of risky inter-temporal choice are all utility-based models. Utility models assume that when making choices participants calculate the utility, or worth, of each option offered and decide based on a comparison of these utilities-i.e., maximizing utility (Vlaev, Chater, Stewart \& Brown, 2011). ${ }^{1}$ The exact way in which these utilities are calculated and compared differs based on the particular model used.

For the purposes of this paper, we do not test the particular assumptions that each risky inter-temporal choice model makes about how utility is calculated or how risk and delay influence each other in choice. For this reason, we do not give our participants choices like those found in row 3 of Table 1 , which require such assumptions to be invoked. Rather, we test the feasibility of incorporating both risky choices and intertemporal choices into a single model. Given that all three currently proposed models are descendants of Prospect Theory, Hyperbolic Discounting, or both, and reduce to variants of these models in the pure risky or inter-temporal choices, we will focus on versions of Hyperbolic Discounting and Prospect Theory.

\section{Prospect theory}

In Prospect Theory, utilities are calculated by transforming objective outcomes, $x$, (e.g., a $\$ 90$ payment), into subjective values, by way of a value function, $v(x)$. Also, the probability of receiving an outcome, $p$, is transformed into a decision weight via a probability weighting function, $w(p)$. For simple gambles with a single non-zero outcome, the utility of an option is the product of these two components, i.e., $U(g)=$ $w(p) \cdot v(x)$.

Value functions are typically assumed to be concave, reflecting a diminishing sensitivity for amounts. We used a power function, $v(x)=x^{a}$, where $a$ is estimated from data, and values between 0 and 1 produce a concave function. Objective probabilities are transformed using S-shaped functions, allowing for an overweighting of small probabilities and underweighting of large probabilities. We used a function

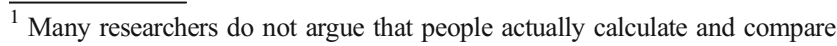
utilities, rather they claim that if people obey certain choice axioms, then they will produce responses that can be described by a utility-based model. The different utility models arise from different assumptions about the underlying choice axioms people are obeying.
} 
proposed by Prelec (1998), $w(p)=e^{-(-\ln p) r}$, where $r$ is estimated from data.

Originally, decisions in Prospect Theory were assumed to be deterministic (Kahneman \& Tversky, 1979). However, because people's behavior is stochastic, modern variants incorporate a choice function, which transforms the difference in utility between options into a probability of choosing the options. Different choice functions have been proposed, we used

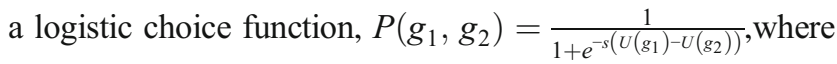
$g_{1}$ and $g_{2}$ are the options being compared, and $s$ is a free parameter estimated from the data.

Prospect Theory, and its derivative, Cumulative Prospect Theory are widely used in the risky choice literature, although different researchers have used different specifications for the value, probability weighting, and choice functions (Kahneman \& Tversky, 1979; Nilsson, Rieskamp \& Wagenmakers, 2011; Rieskamp, 2008; Stott, 2006; Tversky $\&$ Kahneman, 1992). We used the combination of value, probability weighting, and choice functions suggested by Stott (2006) to outperform other versions of Prospect Theory, in terms of fit and parsimony.

\section{Hyperbolic discounting}

Models for making inter-temporal choices are similar and usually involve a value function, discount function, and choice function (Doyle, 2013). The typical Hyperbolic Discounting model uses a hyperbolic discount function, $d(t)=1 /(1+h t)$, where $t$ is the time delay, suggesting diminishing sensitivity to delays, with $h$ being estimated from the data. We used a value function, $v(x)=x^{a}$ and constrain $a>0$. Although it is common to fix $a=1$ in models of delay discounting (Kirby \& Marakovic, 1995; Rachlin, Raineri \& Cross, 1991; Zauberman, Kim, Malkoc \& Bettman, 2009), our investigation directly relates to whether this is a reasonable assumption. Just as in Prospect Theory, these utilities, $U(g)=d(t) \cdot v(x)$, are then fed into a logistic choice function.

\section{Experiment}

We report the result of an experiment in which participants completed both risky decisions and inter-temporal decisions. We fit the choices participants make with four models. One model assumes a single value and choice function for both risky and inter-temporal choices. Another model assumes a separate value and choice function for each set of choices. Two intermediate models test whether one of these functions holds constant and the other varies. We used Bayesian model selection to discriminate between these alternative accounts of risky and inter-temporal choices.

\section{Method}

\section{Participants}

Seventy-two, first-year psychology students from the University of New South Wales participated for course credit. All choices were hypothetical.

\section{Materials}

A total of 130 risky and 130 inter-temporal choices were created. All risky choices involved a choice between two binary gambles. Each gamble involved a probability of gaining some amount of money or else receiving nothing (\$0). The probability of gaining money ranged from 0.05 to 1 in increments of 0.05 , and the amount of money ranged from $\$ 50$ to $\$ 475$ in $\$ 25$ increments. The probability of receiving $\$ 0$ therefore ranged from 0 to 0.95 in increments of 0.05 . Choices were generated by crossing 5 probability levels (very low [0.05$0.2]$, low [0.25-0.4], moderate [0.45-0.6], high [0.65-0.8], very high [0.85-1]) with 6 amount levels (very low [\$50-\$100], low [\$125-\$175], moderately low [\$200-\$250], moderately high [\$275-\$325], high [ $\$ 350-\$ 400]$, very high [\$425-\$475]) for each of the two gambles, yielding 435 unique choice pairings. The specific outcome amount and probability for each option was then determined by drawing a value randomly from the appropriate range. Similar to Rieskamp (2008), we removed all choices in which one option either dominated the other or had twice the Expected Value of the other. This process resulted in 125 choices. Five dominated choices were then added to act as check questions.

To create inter-temporal choices, rather than generate a new choice set, we replaced each probability level in the risky choices with a matching delay, and removed the $\$ 0$ outcomes. Because we used Expected Value to reduce our risky choice set, we decided to match our risky and inter-temporal choice sets by ensuring that the Discounted Value of the intertemporal options approximately matched the Expected Value of their corresponding risky choice option. This required the setting of an exponential discount rate. We set the discount rate by fitting an Exponential Discount function to the mean inter-temporal choice data from the experiments reported in Luckman, Donkin, and Newell (2015), where we had used a similar group of participants and similar ranges of amounts. The fitted rate was 0.053 . The delays we generated were then altered to create the following, easily interpretable lengths: 0 , $1,2,3,4,5,6,8,9,12,13,15,18,21,24,27,30,36,42$, and 54 months. All delays of 12 months and above were split into month and year components when presented to participants.

Choices (130) between a risky option and an inter-temporal option were also created using a similar method; however, these choices were included primarily to provide information 
for a future project and are not of direct interest to this study. An example of one of these Risk vs. Delay choices is given in row 4 of Table 1. Analysis of these choices requires additional assumptions regarding the process for explicit comparison of an option that only contains risk with one that only contains delay. These assumptions are unrelated to the question of interest in this paper.

\section{Procedure}

Participants were told that they would be making choices that involved a mixture of risks and delays. Each choice would be between two options, and there were no correct answers, rather we were interested in their preferences. They also were told to consider each choice independently and that all choices were hypothetical.

The task was split into 3 sections of 130 choices each. For half of the participants, choices were blocked; each section only contained one type of choice (e.g., Risky Choice, Intertemporal choice, or Risk vs. Delay). For the other half of participants, each section contained a mixture of all choice types. The order of choices was randomized within each section for all participants. No differences in preferences were found between these conditions, so we collapse across the blocked and mixed conditions in all subsequent analyses. ${ }^{2}$

\section{Analyses}

Four seminested models were fit to each participant's data. The models differed on whether the value function parameter, $a$, and the choice function parameter, $s$, were constrained to be the same for risky and inter-temporal choices or allowed to differ. These models are referred to as the Common Value and Choice (CvCc), Common Value Separate Choice (CvSc), Separate Value Common Choice (SvCc), and Separate Value Separate Choice (SvSc) models. $\mathrm{Cv}$ models have one $a$ parameter, whereas $\mathrm{Sv}$ models have separate $a$ parameters for risky and inter-temporal choices $\left(a_{R}\right.$ and $\left.a_{I}\right)$. Similarly, Cc models have one $s$ parameter, whereas Sc models have two: one for each choice type $\left(s_{R}\right.$ and $\left.s_{I}\right)$.

\section{Comparison method}

The four models differ in their flexibility/complexity, so they cannot be compared purely based on how well they fit the observed data. If we compared purely based on fit the Separate Values and Choice model would always perform best, because its extra parameters allow it to perfectly mimic the other three models, while also predicting unique patterns.

\footnotetext{
2 The original goal of this manipulation was to see whether participants were more likely to behave consistently, in terms of value and choice functions, when risky and inter-temporal choices were inter-mixed.
}

We used Bayesian model selection via Bayes factors to choose between the competing models, because Bayes factors impose an inherent penalty for model complexity. To obtain Bayes factors, we calculated the marginal likelihood of each model, which is the average likelihood of the data, as weighted by the prior probability of all possible parametrizations of the model.

Bayes factors, therefore, require us to define a prior distribution on the parameters of our models. One way to do this is to base prior distributions on the distribution of parameter values observed in previous experiments. We report the Bayes factors when using the distribution of parameters observed in Luckman, Donkin, and Newell (2015) to inform our prior distributions. We chose the Luckman et al. study, because their three experiments were run on a similar participant pool $(\mathrm{N}=101)$ and involved similar ranges of risks, delays, and amounts to those used here.

The prior distributions for each parameter were obtained by fitting statistical distributions to the observed parameter values from all experiments in Luckman et al. (2015), excluding the most extreme $2 \%$ of values. We used the same set of priors for the discount and decision weight parameters for all four models. The priors for value and choice parameters, however, depended on the particular model being fit. Additionally, rather than drawing $a_{I}$ from a prior distribution, instead we set a prior distribution for the difference between the two value parameters $a_{I}$ and $a_{R}\left(a_{I}-a_{R}\right)$. The full specification of the prior distributions can be found in the Supplementary Materials.

To calculate the marginal likelihood of each model for each individual, we used the brute force method outlined in Vandekerckhove, Matzke and Wagenmakers (2015). This method involves drawing random samples from the prior distributions of each parameter, e.g., $p(a)$. For each sampled set of parameters, $\theta_{t}$, we calculated the probability of the data $p\left(D \mid \theta_{t}\right)$. The marginal likelihood is obtained by averaging over all of the sampled probabilities, $\frac{\sum_{i} p\left(D \mid \theta_{i}\right)}{N}$. We based our marginal likelihoods on $N=$ 600,000 samples for each model for each individual.

\section{Results}

Participants who failed to choose the dominant option on more than one of the five check questions in either choice set were excluded from analysis. Application of this criterion resulted in the exclusion of six participants. Note that the exclusion of these participants does not affect the overall pattern of results.

\section{Group choice}

The left panel of Figure 1 shows the proportion of participants who chose the safer of the two options on each risky choice. The choices have been plotted as a function of their Expected 


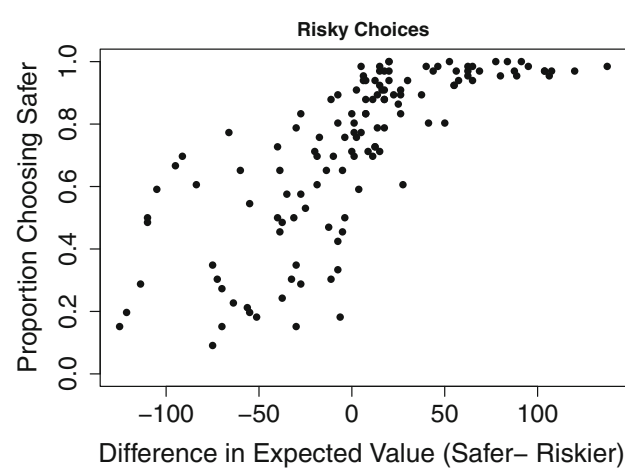

Figure 1 Proportion of participants choosing the sooner or safer option in each choice pairing. The left panel shows the 130 risky choices, and the right shows the 130 inter-temporal choices. Each individual choice pair is

Value. The figure shows that at the group level participants, predictably, chose the safe option less when it had the lower Expected Value and more when it had the higher.

The right panel shows similar results for the inter-temporal choices. Here, the proportions are those who chose the sooner option, plotted as a function of the Discounted Value that we used to generate our choices. Again, participants at a group level made predictable choices: fewer chose the soon option when it had a lower Discounted Value than when it had a higher.

\section{Bayes factors}

We transformed the marginal likelihoods into a posterior model probability for the $j$ th model (assuming each model has an equal prior probability), using $p\left(M_{j} \mid D\right)=\frac{m\left(D \mid M_{j}\right)}{\sum_{k} m\left(D \mid M_{k}\right)}$. Figure 2 plots the posterior model probabilities of the four models for each individual. Because all models had an equal

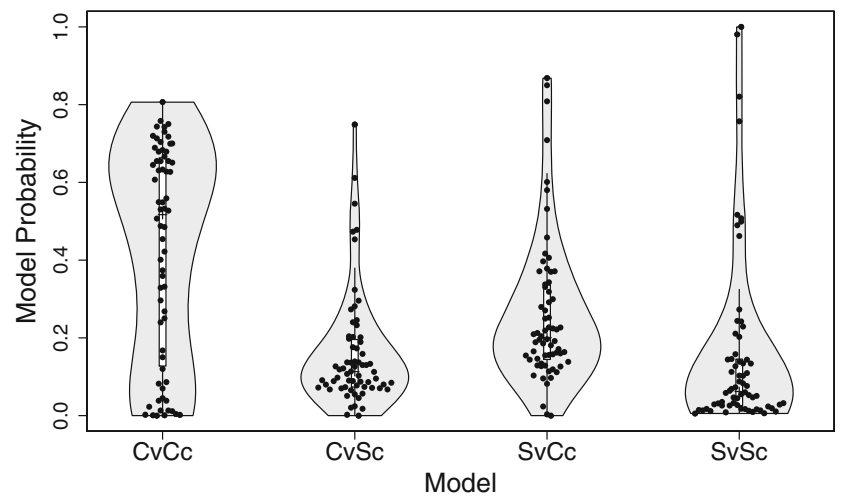

Figure 2 Model probabilities calculated from marginal likelihoods generated using the results of Luckman et al. (2015) to determine prior distributions for each parameter. A prior was placed on the difference between $a_{I}$ and $a_{R}$. Model probabilities are the probability that the given model is the one that generated the data, given the alternatives. Each dot represents an individual participant, with a violin plot overlayed. $\mathrm{CvCc}=$ model assuming Common Value, Common Choice function; $\mathrm{CvSc}=$ Common Value, Separate Choice functions; $\mathrm{SvCc}=$ Separate Value, Common Choice functions; $\mathrm{SvSc}=$ Separate Value, Separate Choice functions

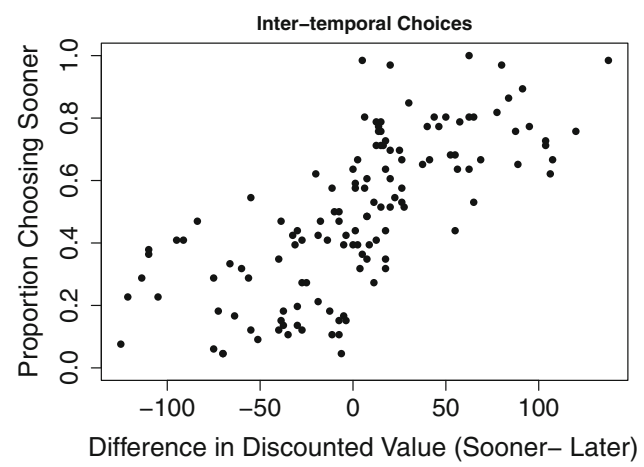

plotted according to the difference in Expected Value (EV) or Discounted Value (DV) between the two options. Negative values on the $\mathrm{x}$ axis are choices where the later/riskier option had a larger DV/EV

prior probability, any model with a probability higher than 0.25 is a model where we have increased evidence or support for this model. For 46 of the 66 participants, the $\mathrm{CvCc}$ model had a probability higher than 0.25 , indicating that the data increased support for this model. For the other three models, evidence for the model generally decreased: only 10 participants had a model probability higher than 0.25 for the $\mathrm{CvSc}$ and SvSc models, whereas 24 participants had a model probability higher than 0.25 for the SvCc model. Therefore, for the majority of participants, the simplest model, which assumes that both the value and choice function are the same for both choice types, was the best fitting. This pattern suggests that most participants behaved consistently across the risky and inter-temporal choice sets, treating amounts in the same way, and calculating utilities in a comparable fashion.

\section{Sensitivity of our results}

Our analysis suggests that a common value and choice function provides the best account of behaviour for most participants. The Online Supplementary to this manuscript reports the results of a number of alternative model selection analyses. In all cases, our conclusions are supported. In fact, the analysis reported provides the least evidence for the common value, common choice model. Included in the supplementary materials, we find support for the simplest model using: 1) an alternative way of setting prior distributions based on the data in Luckman and colleagues (2015); 2) prior distributions based partially on the parameter values reported in Nilsson and colleagues (2011);3) vague prior distributions that are uninformed by previous data; and 4) using Bayesian Information Criterion.

\section{Discussion}

Our results are generally supportive of the notion that behaviour in risky choices and inter-temporal choices can be 
captured in a single model. Regardless of the model selection process, or the prior distributions chosen, for the majority of the 66 participants, a model that had a single choice function and a single value function outperformed those that allowed either, or both, of these functions to differ between risky choice and inter-temporal choices.

\section{Value function}

At first glance, our results appear to conflict with those of Abdellaoui and colleagues (2013), who found that, on average, value functions were concave for risky choice and linear for inter-temporal choice. Somewhat consistent with their results, we observed that the best-fitting value functions in our models, when fit to our data using maximum likelihood estimation, were closer to linear for inter-temporal choices than for risky choices, although still generally concave. Importantly, however, our model-based analysis implies that it is more parsimonious to assume a single value function for both risky and inter-temporal choices.

Our support for a single value function appears to sit uneasily with the finding of opposing impacts of outcome magnitude in risky and inter-temporal choice; a pattern commonly accounted for via changes to the value function. Specifically, the magnitude effect in inter-temporal choice, whereby people appear to have lower discount rates for larger amounts of money, can be captured by the use of an increasingly elastic value function, whereas the peanuts effect in risky choice, whereby people are less tolerant of risk when the amount offered is larger, can be captured by a decreasingly elastic value function (Scholten \& Read, 2014). ${ }^{3}$ Although we did not include a magnitude manipulation in this study, evidence for a single value function is inconsistent with such changes to the value function across choice types. Rather, our results are consistent with accounts of the magnitude effect that suggest discount rates depend upon both amount and delay (Baucells \& Heukamp, 2010; Vincent, 2016) or accounts of the peanuts effect suggesting that probability weighting depends upon both probability and amount (Myerson et al., 2011, Stevens, 1961). These alternative explanations also are consistent with findings reported in choices between risks and delays (Table 1, row 4), which demonstrated both magnitude and peanuts effects simultaneously and cannot be captured by elasticity in the value function (Baucells \& Heukamp, 2010; Luckman, Donkin \& Newell, in press). Nevertheless, future studies will need to test explicitly whether a single value

\footnotetext{
3 The elasticity of the value function refers to the ratio between the proportional change in an outcomes subjective value, $v(x)$, and the proportional change in its objective value, $x$. The power function we used has constant elasticity, because this ratio is the same regardless of the value of $x$. If this ratio is smaller (larger) for large values of $x$ than for small values of $x$, the function is decreasingly (increasingly) elastic.
}

function applied to both risky and inter-temporal choice, with appropriately modified discount rate and probability weighting functions, outperforms separate value functions with differing elasticity properties in situations where outcome magnitude is systematically manipulated.

From a broader theoretical perspective, using a standard Economics-based interpretation of the value function, a single value function suggests that utility calculations may be constant across risky and inter-temporal choice domains, perhaps reflecting a stable internal construct. Alternatively, our result is consistent with the interpretation of the value function as reflecting psychophysical scaling, which one would expect to be stable across risky and inter-temporal choice domains (Myerson, et al. 2011).

\section{Choice function}

Unlike the value function, to our knowledge our results regarding the choice function are the first to explore whether risky choices and inter-temporal choices are made using the same types of "evidence." Our results suggest a common choice function, which has interesting implications for the development of models of risky inter-temporal choice. One could argue that a common value function may be due to a common process, such as psychophysical scaling, which is unrelated to the particular choice type. As such, a common choice function may be stronger evidence that risky and intertemporal choice types are special cases of a common decision process. Future work may examine whether choice functions are constant in choices that include options varying in both risk and delay.

\section{Future work}

Our results provide preliminary evidence for the notion that risky choices and inter-temporal choices are a special case of risky inter-temporal choices. This assumption is an implicit feature of the three existing models of risky inter-temporal choice, the Probability and Time Trade-off model, the Multiplicative Hyperbolic Discounting model, and the Hyperbolic Discounting model (Baucells \& Heukamp, 2010; Vanderveldt et al., 2015; Yi et al., 2006). However, we do not claim that this is the only, nor definitive, way to test this assumption.

An alternative approach would be to use the method we have employed but applied to a dataset that includes risky choices, inter-temporal choices, and risky inter-temporal choices (Table 1, rows 1, 2, and 3). The disadvantage of such an approach is that it requires additional assumptions about the relationship between risk and delay in risky inter-temporal choice, which the current analysis does not. If these additional 
assumptions are wrong, it undermines our ability to test the underlying assumption of a single model. Given that the existing models of risky inter-temporal choice do not agree on these assumptions (Baucells \& Heukamp, 2010, 2012; Vanderveldt et al., 2015; Yi et al., 2006), we chose to focus on pure risky and inter-temporal choices to avoid committing to one particular model of risky inter-temporal choice and its associated assumptions. However, future work, following testing of these additional assumptions, should assess whether such models can account for the full range of pure risky, pure inter-temporal, and risky inter-temporal choices using single value and choice functions.

\section{Boundary conditions}

Also of future interest will be the boundary conditions limiting when choices involving risk and inter-temporal features operate under a single, common mechanism. One of these boundary conditions may already have been identified using a nonmodel-based approach. Luckman et al. (in press) used participants' responses in pure risky and pure inter-temporal choices to predict preferences in a choice between an outcome that is risky and the same outcome with a delay. This is similar to the Risk vs. Delay choice in Table 1 (row 4), but with the outcome amount matched across options (i.e., both \$150). Luckman et al. found that preferences did not match those predicted by a common utility-based mechanism underlying the three types of choice. Therefore, this particular subtype of risky inter-temporal choice, where only one option involves risk, the other option involves delay, and both options have the same outcome, may present a boundary situation where a common mechanism does not hold.

Another boundary condition might be situations that involve losses. Unlike risky choices, there is evidence that people are unwilling to delay losses and may even consider a delayed loss to be worse than an immediate loss (Hardisty, Appelt \& Weber, 2013). Such a shift in behaviour may be difficult to reconcile within a single model framework.

\section{Conclusions}

Overall, we find preliminary support for a unifying model of risky, inter-temporal, and risky inter-temporal choices. Models with a single value and choice function could account for both pure risky and pure inter-temporal choices. Our results are consistent with any model that implicitly assumes that risky or inter-temporal choices are special cases of risky inter-temporal choices.

Acknowledgements BRN was supported by an Australian Research Council Future Fellowship (FT110100151), and an ARC Discovery
Project (DP 140101145). CD was supported by an Australian Research Council DECRA Fellowship (DE130100129). AL was supported by a UNSW PhD Scholarship.

\section{References}

Abdellaoui, M., Bleichrodt, H., l'Haridon, O., \& Paraschiv, C. (2013). Is there one unifying concept of utility? an experimental comparison of utility under risk and utility over time. Management Science, 59(9), 2153-2169. doi:10.1287/mnsc. 1120.1690

Baucells, M., \& Heukamp, F. H. (2010). Common ratio using delay. Theory and Decision, 68(1-2), 149-158. doi:10.1007/s11238-0089130-2

Baucells, M., \& Heukamp, F. H. (2012). Probability and time trade-off. Management Science, 58(4), 831-842. doi:10.1287/mnsc.1110. 1450

Doyle, J. R. (2013). Survey of time preference, delay discounting models. Judgment and Decision Making, 8(2), 116-135.

Kahneman, D., \& Tversky, A. (1979). Prospect theory: an analysis of decision under risk. Econometrica, 47, 263-291.

Kirby, K. N., \& Marakovic, N. N. (1995). Modeling myopic decisions evidence for hyperbolic delay-discounting within-subjects and amounts. Organizational Behavior and Human Decision Processes, 64(1), 22-30. doi:10.1006/obhd.1995.1086

Luckman, A., Donkin, C., \& Newell, B. R. (in press). People wait longer when the alternative is risky: The relation between preferences in risky and inter-temporal choice. Journal of Behavioral Decision Making. doi:10.1002/bdm.2025

Luckman, A., Donkin, C., \& Newell, B. R. (2015). Exploring the concept of utility: Are separate value functions required for risky and intertemporal choice? In D. C. Noelle, R. Dale, A. S. Warlaumont, J. Yoshimi, T. Matlock, C. D. Jennings, \& P. P. Maglio (Eds.), Proceedings of the 37th Annual Conference of the Cognitive Science Society (pp. 1440-1446). Austin: Cognitive Science Society.

Myerson, J., Green, L., Hanson, J. S., Holt, D. D., \& Estle, S. J. (2003). Discounting delayed and probabilistic rewards: Processes and traits. Journal of Economic Psychology, 24(5), 619-635. doi:10.1016/ s0167-4870(03)00005-9

Myerson, J., Green, L., \& Morris, J. (2011). Modeling the effect of reward amount on probability discounting. Journal of the Experimental Analysis of Behavior, 95(2), 175-187. doi:10.1901/jeab.2011.95175

Nilsson, H., Rieskamp, J., \& Wagenmakers, E. J. (2011). Hierarchical Bayesian parameter estimation for cumulative prospect theory. Journal of Mathematical Psychology, 55(1), 84-93. doi:10.1016/j. jmp.2010.08.006

Prelec, D. (1998). The probability weighting function. Econometrica, 66(3), 497-527. doi:10.2307/2998573

Rachlin, H., Raineri, A., \& Cross, D. (1991). Subjective probability and delay. Journal of the Experimental Analysis of Behavior, 55(2), 233 244. doi:10.1901/jeab.1991.55-233

Rieskamp, J. (2008). The probabilistic nature of preferential choice. Journal of Experimental Psychology: Learning, Memory, and Cognition, 34(6), 1446-1465. doi:10.1037/a0013646

Scholten, M., \& Read, D. (2014). Prospect theory and the "forgotten" fourfold pattern of risk preferences. Journal of Risk and Uncertainty, 48(1), 67-83. doi:10.1007/s11166-014-9183-2

Stevens, S. S. (1961). To honor Fechner and repeal his law: A power function, not a log function, describes the operating characteristic of a sensory system. Science, 133(3446), 80-86. doi:10.1126/ science.133.3446.80 
Stott, H. P. (2006). Cumulative prospect theory's functional menagerie. Journal of Risk and Uncertainty, 32(2), 101-130. doi:10.1007/ s11166-006-8289-6

Tversky, A., \& Kahneman, D. (1992). Advances in prospect theory: Cumulative representation of uncertainty. Journal of Risk and Uncertainty, 5(4), 297-323. doi:10.1007/Bf00122574

Vandekerckhove, J., Matzke, D., \& Wagenmakers, E. J. (2015). Model comparison and the principle of parsimony. In J. R. Busemeyer, J. Townsend, Z. J. Wang, \& A. Aidels (Eds.), Oxford handbook of computational and mathematical psychology (pp. 300-319). Oxford: Oxford University Press.

Vanderveldt, A., Green, L., \& Myerson, J. (2015). Discounting of monetary rewards that are both delayed and probabilistic: Delay and probability combine multiplicatively, not additively. Journal of
Experimental Psychology: Learning, Memory, and Cognition, 41(1), 148-162. doi:10.1037/xlm0000029

Vincent, B. T. (2016). Hierarchical Bayesian estimation and hypothesis testing for delay discounting tasks. Behavior Research Methods, 48(4), 1608-1620. doi:10.3758/s13428-015-0672-2

Vlaev, I., Chater, N., Stewart, N., \& Brown, G. D. A. (2011). Does the brain calculate value? Trends in Cognitive Sciences, 15(11), 546554. doi:10.1016/j.tics.2011.09.008

Yi, R., de la Piedad, X., \& Bickel, W. K. (2006). The combined effects of delay and probability in discounting. Behavioural Processes, 73(2), 149-155. doi:10.1016/j.beproc.2006.05.001

Zauberman, G., Kim, B. K., Malkoc, S. A., \& Bettman, J. R. (2009). Discounting time and time discounting: subjective time perception and intertemporal preferences. Journal of Marketing Research, $46(4), 543-556$. 